\title{
Le contenu et la valeur juridique du principe de laïcité
}

Notes sous : Conseil constitutionnel, décision $n^{\circ} 2012-297$ QPC du 21 février 2013, Association pour la promotion et l'expansion de la laïcité [Traitement des pasteurs des églises consistoriales dans les départements du Bas-Rhin, du Haut-Rhin et de la Moselle] The content and legal force of the principle of secularism. Notes on: Constitutional Council, decision No. 2012-297 QPC of 21 February 2013, Association for the promotion and expansion of secularism [Treatment of pastors from consistorial churches in the Bas-Rhin, Haut-Rhin and Moselle departments]

\section{Henri Bouillon}

\section{(2) OpenEdition}

\section{Journals}

Édition électronique

URL : http://journals.openedition.org/add/697

DOI : $10.4000 /$ add. 697

ISSN : 2606-1988

Éditeur

Presses universitaires de Rouen et du Havre

Édition imprimée

Date de publication : 1 mai 2014

Pagination : 09-31

ISBN : 979-10-240-0161-6

ISSN : 1955-0855

Référence électronique

Henri Bouillon, « Le contenu et la valeur juridique du principe de laïcité », Les Annales de droit [En ligne], 8| 2014, mis en ligne le 14 mars 2018, consulté le 10 décembre 2020. URL : http:// journals.openedition.org/add/697 ; DOI : https://doi.org/10.4000/add.697 


\title{
Le contenu et la valeur juridique du principe de laïcité ${ }^{1}$
}

\author{
Notes sous: Conseil constitutionnel, \\ décision $n^{\circ}$ 2012-297 QPC du 21 février 2013, \\ Association pour la promotion et l'expansion de la laïcité \\ [Traitement des pasteurs des églises consistoriales dans les \\ départements du Bas-Rhin, du Haut-Rhin et de la Moselle]
}

Henri BouILLoN

L'article $1^{\text {er }}$ de la Constitution proclame, sans définir la notion, que la République française est laïque. Si l'assertion ne compte guère de détracteurs, il n'est pas certain qu'elle soit aussi claire que l'usage courant dont elle fait l'objet le laisse supposer. La procédure de question prioritaire de constitutionnalité (QPC) a été l'occasion pour le Conseil constitutionnel, interprète privilégié de la Constitution, d'en venir clarifier l'article $1^{\text {er }}$ en lui conférant une signification et une texture juridique plus palpable.

Le 19 décembre 2012, le greffe du Conseil constitutionnel recevait la transmission d'une QPC par le Conseil d'État ${ }^{2}$, relativement à la constitutionnalité de l'article VII des articles organiques applicables aux cultes protestants de la loi du 18 germinal an X. Selon l'article, « il sera pourvu au traitement des pasteurs des églises consistoriales, bien entendu qu'on imputera sur ce traitement les biens que ces églises possèdent, et le produit des oblations établies par l'usage ou par des règlements ». Est ainsi organisée la rémunération, par l'État, des ministres du culte, protestants en l'occurrence. Or, l'Association pour la promotion et l'expansion de la laïcité (APPEL), à l'origine de la requête, soutient que cette disposition porte atteinte au principe constitutionnel de laïcité : l'association « fait valoir que la règle de non-subventionnement des cultes et le principe

1. Nous tenons à remercier chaleureusement Alexandre Leconte pour les nombreuses réflexions qu'il a bien voulu faire à propos de la première version de cet article et pour l'intérêt qu'il a manifesté pour ce travail.

2. CE, 19 décembre 2012, Association pour la promotion et l'expansion de la laïcité (APPEL), $\mathrm{n}^{\text {os }} 360724$ et 360725 . 
de non-reconnaissance des cultes, qui résultent du principe de laïcité, font interdiction aux pouvoirs publics de financer l'exercice du culte et d'accorder un statut ou un soutien public à des cultes déterminés » (considérant 2).

1) Par la décision APPEL du 21 février $2013^{3}$, le Conseil constitutionnel a rejeté les prétentions de l'APPEL et a jugé conforme à la Constitution l'article précité. Le raisonnement qui mène à cette solution est toutefois déroutant. Adoptant la forme du syllogisme, ce raisonnement se développe en trois phases:

En premier lieu, le Conseil constitutionnel expose la disposition qui lui est déférée (considérant 1) ainsi que les motifs pour lesquels ces dispositions concordataires sont restées en vigueur dans les trois départements du Bas-Rhin, du Haut-Rhin et de la Moselle (considérants 3 et 4).

Dans un deuxième temps, le Conseil éclaircit la norme de référence, au regard de laquelle il va juger de la constitutionnalité de la loi. Cette norme de référence est le principe constitutionnel de laïcité. Le Conseil définit ce qu'il estime être ses composantes essentielles. Il juge « que le principe de laïcité figure au nombre des droits et libertés que la Constitution garantit; qu'il en résulte la neutralité de l'État; qu'il en résulte également que la République ne reconnaît aucun culte; que le principe de laïcité impose notamment le respect de toutes les croyances, l'égalité de tous les citoyens devant la loi sans distinction de religion et que la République garantisse le libre exercice des cultes; qu'il implique que celle-ci ne salarie aucun culte» (considérant 5 ).

Dans une troisième phase, la norme de référence étant éclaircie, le Conseil va la confronter avec la disposition législative et se prononcer sur leur rapport, c'est-à-dire sur leur conformité ou leur non-conformité. Le problème soulevé par ce rapport est aussi aisé à dégager qu'il est difficile à résoudre. On peut le formuler de la façon suivante: l'existence d'une rémunération des ministres du culte par l'État est-elle une atteinte au principe constitutionnel de laïcité ? La conformité des deux normes parait a priori douteuse : puisque, aux dires du Conseil lui-même, le principe de laïcité comprend l'interdiction de salarier les cultes, l'article contesté de la loi de l'an $\mathrm{X}$, qui permet la rémunération

3. CC, décision $n^{0}$ 2012-297 QPC du 21 février 2013, APPEL [Traitement des pasteurs des églises consistoriales dans les départements du Bas-Rhin, du Haut-Rhin et de la Moselle]; JCP A, 2013, $\mathrm{n}^{\circ}$ 12, act. 243, note Jean-François Amédro ; JCP A, 2013, $\mathrm{n}^{\circ} 16$, p. 2108, note Franck Laffaille; $A J D A$, 2013, p. 1108, note Elsa Forey; Constitutions, 2013, $\mathrm{n}^{\circ}$ 2, p. 174, note Philippe Lutton; Droit administratif, 2013, $\mathrm{n}^{\circ} 8$, étude 14, note Frédérique de La Morena. 
des ministres du culte par l'État, est en opposition manifeste avec ce principe. Pourtant, le Conseil admet la conformité de cette disposition législative à la Constitution. La seule explication qu'il en donne figure au considérant 6 : «il ressort tant des travaux préparatoires du projet de la Constitution du 27 octobre 1946 relatifs à son article $1^{\text {er }}$ que de ceux du projet de la Constitution du 4 octobre 1958 qui a repris la même disposition, qu'en proclamant que la France est une "République... laïque", la Constitution n'a pas pour autant entendu remettre en cause les dispositions législatives ou réglementaires particulières applicables dans plusieurs parties du territoire de la République lors de l'entrée en vigueur de la Constitution et relatives à l'organisation de certains cultes et, notamment, à la rémunération de ministres du culte. » La volonté des constituants de 1946 et 1958 assure ainsi la conformité des dispositions législatives locales avec le principe constitutionnel de laïcité.

2) La thèse que ces quelques réflexions entendent proposer peut être résumée en quelques assertions:

Dans cette décision, le Conseil constitutionnel conclut à la conformité $\mathrm{du}$ droit des cultes alsacien-mosellan avec l'article $1^{\mathrm{er}}$ de la Constitution. Nous tiendrons cette conclusion pour acquise. Nos critiques se concentreront sur le raisonnement qui y aboutit et qui est étayé par une argumentation bien faible. Mentionné au considérant 6, l'argument unique de la décision repose sur la volonté des constituants de 1946 et 1958. Cet argument est insatisfaisant en raison de l'ombre qu'il laisse s'appesantir sur le cœur même du problème, soigneusement éludé par le juge constitutionnel : la conformité de la rémunération des ministres du culte par l'État avec le principe constitutionnel de laïcité (1). Ce n'est donc pas la conclusion, mais bien le raisonnement du Conseil constitutionnel qui est hautement contestable.

Pour détecter l'origine de cette déficience, on doit remonter à la deuxième phase du raisonnement, c'est-à-dire à la définition de la laïcité énoncée au considérant 5 de la décision. Cette définition engendre à l'évidence la non-conformité de la norme de référence et de la norme contrôlée: si la laïcité interdit de salarier les cultes et puisque la rémunération des ministres du culte s'apparente sans conteste à un tel financement, les dispositions de l'article $1^{\text {er }}$ de la Constitution et de la loi de l'an X sont immanquablement inconciliables. Or, on voudrait ici montrer qu'une autre interprétation du principe constitutionnel de laïcité, plus en accord avec le droit positif, aurait pu assurer plus correctement sa conformité avec le droit alsacien-mosellan et donner un fondement plus solide au maintien de ce droit local (2). Car l'enjeu 
de cette question est de justifier l'existence du droit des cultes alsacienmosellan: et la justification donnée par le Conseil nous paraissant bien faible, il nous semble qu'il faille, non pas remettre en cause la constitutionnalité du droit local, mais bien la définition de la laïcité retenue par le Conseil constitutionnel.

\section{La faible justification de la conformité de la laïcité avec la rémunération locale des ministres du culte par l'État}

Après un long mutisme du juge constitutionnel, le droit alsacienmosellan a récemment fait l'objet de plusieurs décisions d'importance. Celles-ci permirent d'asseoir constitutionnellement l'existence de ce droit local par la découverte d'un PFRLR (1.1). La présente décision portait plus spécifiquement sur le droit local des cultes et sur son respect du principe constitutionnel de laïcité. Or le Conseil constitutionnel n'adopte pas un raisonnement identique à celui tenu dans ses décisions précédentes. Pour juger de la constitutionnalité de la rémunération des ministres du culte par l'État, il effectue un contrôle de l'abrogation implicite qui, appuyé sur la volonté des constituants (1.2), appelle de nombreuses critiques (1.3).

\subsection{L'assise constitutionnelle du droit alsacien-mosellan dans la jurisprudence constitutionnelle antérieure}

Jusqu'en 2011, le juge constitutionnel avait, lorsqu'était invoquée devant lui l'inconstitutionnalité de la législation spécifique aux départements d'Alsace et à la Moselle, évacué rapidement la question, l'abandonnant aux bons soins du législateur. Il avait pu juger « que la particularité de la législation locale sur les associations ainsi relevée ne procède pas de la loi soumise à l'examen du Conseil constitutionnel et ne saurait, du seul fait de ses conséquences, entacher celle-ci d'inconstitutionnalité; qu'il appartiendra au législateur d'apprécier s'il y a lieu de réparer cette anomalie $^{4} »$. Les choses auraient pu évoluer avec la jurisprudence État d'urgence en Nouvelle-Calédonie, qui permet de contrôler une loi déjà promulguée "à l'occasion de l'examen de dispositions législatives qui la modifient, la complètent ou affectent son domaine ${ }^{5}$ ». Mais le Conseil n'était pas sorti de sa réserve, considérant «que le particularisme du

4. CC, décision $n^{0}$ 82-153 DC du 14 janvier 1983, Loi relative au statut général des fonctionnaires, considérant 21.

5. CC, décision $\mathrm{n}^{0}$ 85-187 DC du 25 janvier 1985, Loi relative à l'état d'urgence en Nouvelle-Calédonie et dépendances, considérant 10. 
droit local ne procède pas de l'article $1^{\text {er }}$ de la loi soumise à l'examen du Conseil constitutionnel; que la loi déférée ne modifie, ne complète ni n'affecte le domaine d'intervention de la loi du 19 avril 1908; que la mention de ce dernier texte ne saurait, en tout état de cause, entacher la loi présentement examinée d'inconstitutionnalité ${ }^{\star}$.

La QPC contraignit le Conseil constitutionnel à se montrer plus loquace. La constitutionnalité du droit alsacien-mosellan fut abordée frontalement par la décision Société Somodia ${ }^{7}$ du 5 août 2011. La société requérante affirmait que l'interdiction du travail le dimanche, qui ne demeure qu'en Alsace et en Moselle, méconnaissait le principe d'égalité devant la loi. Le Conseil ne fut pas de cet avis. Il affirma que «la législation républicaine antérieure à l'entrée en vigueur de la Constitution de 1946 a consacré le principe selon lequel, tant qu'elles n'ont pas été remplacées par les dispositions de droit commun ou harmonisées avec elles, des dispositions législatives et réglementaires particulières aux départements du Bas-Rhin, du Haut-Rhin et de la Moselle peuvent demeurer en vigueur; qu'à défaut de leur abrogation ou de leur harmonisation avec le droit commun, ces dispositions particulières ne peuvent être aménagées que dans la mesure où les différences de traitement qui en résultent ne sont pas accrues et que leur champ d'application n'est pas élargi; que telle est la portée du principe fondamental reconnu par les lois de la République en matière de dispositions particulières applicables dans les trois départements dont il s'agit; que ce principe doit aussi être concilié avec les autres exigences constitutionnelles ${ }^{8}$ ».

Bien qu'elle ne soit pas l'objet de la présente étude, cette décision mérite que l'on s'y arrête. Son apport fondamental est de consacrer un Principe fondamental reconnu par les lois de la République (PFRLR), qui protège la législation locale en conférant à son existence un rang constitutionnel. Son effet immédiat est de soustraire à la critique le particularisme du droit applicable dans les trois départements d'AlsaceMoselle au regard du principe d'égalité des citoyens devant la loi.

6. CC, décision $\mathrm{n}^{0}$ 91-299 DC du 2 août 1991, Loi relative au congé de représentation en faveur des associations et des mutuelles et au contrôle des comptes des organismes faisant appel à la générosité publique, considérant 5.

7. CC, décision $\mathrm{n}^{\circ}$ 2011-157 QPC du 5 août 2011, Société Somodia [Interdiction du travail le dimanche en Alsace-Moselle]. JCP G, 2011, $\mathrm{n}^{\circ}$ 47, p. 1290, note Michel Verpeaux; JCP A, 2011, $\mathrm{n}^{\circ}$ 42, p. 2319, note Nadi Abi Rached; RFDA, 2011, $\mathrm{n}^{\circ} 6$, p. 1209, note Agnès Roblot-Troizier; $A J D A, 2012, \mathrm{n}^{\circ}$ 6, p. 331, note Anne Jennequin; Recueil Dalloz, 2012, $\mathrm{n}^{0}$ 16, p. 1047, note Patricia Hennion-Jacquet; RDT, 2011, p. 574, note Éric Sander; RFDA, 2012, $\mathrm{n}^{\mathrm{o}}$ 1, p. 131, note Jean-Marie Woerhling.

8. CC, décision $\mathrm{n}^{\circ}$ 2011-157 QPC du 5 août 2011, Société Somodia, considérant 4. 
Toutefois, si «le droit local alsacien-mosellan est protégé dans son existence $^{9}$ ", ce PFRLR ne justifie pas un particularisme illimité. Le Conseil pose deux restrictions qui encadrent la spécificité du droit local et empêchent de lui décerner, par avance, un brevet de constitutionnalité.

Premièrement, il n'existe pas de garantie constitutionnelle au maintien des dispositions législatives ou réglementaires locales. Le Parlement ou les titulaires du pouvoir réglementaire, selon que sont concernées les matières de l'article 34 ou 37 de la Constitution, peuvent à tout moment modifier ou abroger des dispositions locales pour les remplacer par les dispositions de droit commun ou les harmoniser avec celles-ci. Le PFRLR dégagé par le Conseil n'a pas vocation à cristalliser le droit local. Il ne restreint en rien le pouvoir dont disposent les autorités publiques pour procéder à sa modification, à condition toutefois que le particularisme ne soit pas amplifié. En effet, le Conseil interdit formellement un accroissement du particularisme existant. Toute modification du droit local ne peut s'effectuer qu'en vue d'une uniformisation avec le droit national. "La décision Société Somodia n'interdit nullement au législateur d'abroger le droit local, et donc d'harmoniser l'ensemble du droit. Si l'existence d'un droit local n'est pas contraire à la Constitution, sa disparition ne l'est pas non plus ${ }^{10}$.»

Deuxièmement, en soulignant que «ce principe doit aussi être concilié avec les autres exigences constitutionnelles », le Conseil entend ne pas priver d'effectivité les autres normes constitutionnelles. Celles-ci demeurent applicables et doivent être conciliées avec ce PFRLR. Celui-ci ne peut justifier toute dérogation aux principes constitutionnels. S’il permet d'empêcher une inconstitutionnalité globale du droit local, des dispositions locales peuvent être individuellement déclarées inconstitutionnelles si elles méconnaissent d'autres principes intégrés dans le bloc de constitutionnalité. "La reconnaissance comme PFRLR du maintien du droit local ne constitue pas un brevet de constitutionnalité pour le contenu de celui-ci, pas davantage une libération du contrôle de constitutionnalité, ni non plus une garantie de son maintien ${ }^{11}$. " Dans la décision Société Somodia, le Conseil s'est ainsi penché sur le respect de la liberté d'entreprendre, pour juger que la disposition législative en

9. Anne Jennequin, «Le contrôle du droit local alsacien-mosellan au regard des droits et libertés constitutionnellement garantis ", AJDA, 2012, p. 331.

10. Fabien Hoffmann, "Le droit alsacien-mosellan au crible de la QPC », Droit administratif, $\mathrm{n}^{\circ} 2,2013$, commentaire 12.

11. Jean-Marie Woehrling, "La décision du Conseil constitutionnel sur le droit local alsacien mosellan: consécration ou restriction? Les difficultés d'élaboration d'un cadre constitutionnel pour une territorialisation du droit », RFDA, 2012, p. 131. 
cause ne lui portait pas atteinte. De même, faisant application de ce PFRLR, la décision Consorts G. $^{12}$ affirme qu'une disposition régissant le droit des successions alsacien-mosellan ne méconnaît pas le principe constitutionnel d'égalité des cohéritiers. Dans ces deux hypothèses, le Conseil n'écarte pas les normes constitutionnelles au seul profit du PFRLR dégagé le 5 août 2011 : il n'admet la constitutionnalité des dispositions législatives qu'après s'être assuré de leur compatibilité avec les autres principes constitutionnels. En outre, la décision M. Christian $S^{13}$ du 30 novembre 2012 illustre la possibilité de censurer individuellement certaines dispositions du droit local. Le Conseil constitutionnel y juge inconstitutionnelles les dispositions législatives applicables dans les départements du Haut-Rhin, du Bas-Rhin et de la Moselle, puisque ces dispositions, en imposant à certaines professions artisanales de s'affilier à des corporations, méconnaissaient la liberté d'entreprendre.

Dans sa décision $A P P E L$, la question soulevée était celle de la conformité des dispositions de la loi de l'an X avec le principe constitutionnel de laïcité. Pour solutionner ce problème, le Conseil n'emploie pas à nouveau ce PFRLR. Il préfère effectuer un contrôle de l'abrogation implicite, appuyé sur la volonté des constituants.

\subsection{Un contrôle de l'abrogation implicite, appuyé sur la volonté des constituants}

La décision APPEL du 21 février 2013 s'appuie sur les travaux préparatoires des constituants de 1946 et 1958 pour affirmer leur volonté de ne pas remettre en cause les dispositions du droit local en matière de culte. Le Conseil constitutionnel paraît faire un contrôle de l'abrogation implicite, mais d'un genre très particulier: son objectif est de montrer que l'entrée en vigueur de la Constitution de 1946, et du principe de laïcité qu'elle consacre, n'a pas abrogé implicitement les dispositions législatives antérieures qui, en Alsace-Moselle, pourraient lui être contraires.

Le contrôle de l'abrogation implicite n'a, en lui-même, rien d'exceptionnel (dans son principe, si ce n'est dans sa fréquence) : il permet de s'assurer qu'une norme n'a pas été implicitement abrogée par l'adoption

12. CC, décision $\mathrm{n}^{0}$ 2012-274 QPC du 28 septembre 2012, Consorts G. [Calcul de l'indemnité de réduction due par le donataire ou le légataire d'une exploitation agricole en Alsace-Moselle], considérants 6 et 7 .

13. CC, décision $\mathrm{n}^{\mathrm{o}}$ 2012-285 QPC du 30 novembre 2012, M. Christian S. [Obligation d'affiliation à une corporation d'artisans en Alsace-Moselle]; Fabien Hoffmann, art. cité. 
d'une norme nouvelle qui, tout en lui étant supérieure ou égale, lui est contraire. Un tel contrôle a par exemple permis au Conseil d'État de constater l'abrogation tacite de certaines dispositions locales, relatives à la constitution de congrégations religieuses, qui étaient contraires au principe d'égalité entre les sexes ${ }^{14}$. De même, le Conseil d'État a, dans son arrêt $S N E S^{15}$, usé de cette méthode lorsqu'il fut saisi d'un recours pour excès de pouvoir contre un arrêté du ministre de l'Éducation nationale, adopté notamment sur le fondement des lois du 17 octobre 1919 et du $1^{\text {er }}$ juin 1924. Il se posait au juge administratif la question suivante: ces dispositions législatives, qui maintiennent en vigueur la législation cultuelle d'Alsace-Moselle après le retour de ces départements dans le giron français, avaient-elles été implicitement abrogées par les Constitutions de 1946 et 1958, qui consacrent le principe de laïcité? En raison du manque de clarté de ce principe, le Conseil d'État a rejeté l'abrogation implicite.

C'est un semblable raisonnement que tient ici le Conseil constitutionnel : il s'interroge sur le point de savoir si la consécration constitutionnelle du principe de laïcité peut entraîner l'inconstitutionnalité des dispositions législatives antérieures qui lui sont déférées. Et pour affirmer que la Constitution n'a pas abrogé implicitement les dispositions locales, le Conseil constate que les travaux préparatoires du constituant entendaient laisser subsister ce particularisme local.

Le contrôle du Conseil est néanmoins d'un genre particulier. La technique de l'abrogation implicite consiste à analyser le caractère conciliable ou inconciliable d'une disposition textuelle avec des dispositions postérieures qui lui sont, dans la hiérarchie des normes, supérieures ou égales. Par cette technique, est ainsi abrogée la loi dont le contenu est « inconciliable avec un texte qui lui est postérieur, que celui-ci ait une valeur législative ou constitutionnelle ${ }^{16} »$. Mais ce n'est point ainsi que procède ici le juge constitutionnel. Pour reconnaître cette constitutionnalité, l'argument exposé par le considérant 6 ne repose pas sur le caractère conciliable de la loi avec le principe de laïcité. Il s'appuie sur la volonté des constituants de 1946 et 1958 de ne pas remettre en

14. CE, avis, sect. de l'intérieur, 16 novembre $1993, \mathrm{n}^{\circ} 355-149$.

15. CE, 6 avril 2001, Syndicat national des enseignements du second degré, $\mathrm{n}^{\mathrm{os}} 219379$, 221699 et 221700; AJDA, 2002, p. 63, note Bernard Toulemonde; $R D L, 2001$, $\mathrm{n}^{\mathrm{o}}$ 33, p. 56, note Jean-Marie Woehrling; Revue de droit canonique, t. LII/2, p. 373, conclusion Emmanuelle Mignon.

16. CE, réf., 21 novembre 2005, Jean-Charles Boisvert, ${ }^{\circ} 287217$. 
cause le régime des cultes établi en Alsace-Moselle. Il n'y a pas abrogation implicite parce que les constituants ne l'ont pas voulu.

Or ce raisonnement est insatisfaisant et appelle une sévère appréciation critique.

\subsection{Appréciation critique du raisonnement tenu par le Conseil constitutionnel}

L'argumentation du Conseil constitutionnel est bien faible pour justifier une décision d'une telle importance, dont l'enjeu n'est rien de moins que l'existence du droit local des cultes. En effet, cette argumentation ne justifie pas l'entorse réalisée au principe de laïcité, tel que le Conseil l'a lui-même cerné, et ce pour plusieurs raisons. Le débat pourrait être porté sur le terrain de savoir comment la Constitution doit être interprétée: doit-elle être interprétée par référence à l'esprit de ses pères fondateurs ou, au contraire, l'interprétation doit-elle être évolutive et adapter la Constitution aux changements de circonstances? Mais cette question théorique, que l'on ne peut manquer d'avoir à l'esprit, ne saurait être développée ici, malgré son indéniable intérêt. D’autres arguments doivent nous retenir davantage.

1) Tout d'abord, compte tenu de la matière concernée par la décision, il semble contestable d'exclure le PFRLR consacré par la décision Société Somodia. De valeur constitutionnelle, il serait plus propre à protéger la loi en cause que la seule intention des Constituants. On comprend cependant pourquoi le Conseil a privilégié cette méthode et n'a pas fait usage du PFRLR dégagé précédemment. En effet, la seconde limite à l'application de ce PFRLR est la nécessité de le concilier avec les autres exigences constitutionnelles, et notamment les droits et libertés que la Constitution garantit. L'appliquer en l'espèce aurait nécessité de le concilier avec le principe de laïcité invoqué par les requérants et qui «figure au nombre des droits et libertés que la Constitution garantit» (considérant 5). Or, la définition que le juge donne ici de la laïcité rend toute conciliation impossible. La laïcité, telle que définie par le Conseil constitutionnel, interdit de salarier les cultes, alors que la loi de l'an $\mathrm{X}$ admet la rémunération de certains ministres du culte : la contradiction est manifeste et irréductible; l'application d'un principe exclut celle de l'autre. Si bien qu'appliquer ce PFRLR aurait imposé de justifier sa conciliation avec le principe de laïcité, ce qui n'était de toute évidence pas dans les intentions du juge constitutionnel.

2) Ensuite, d'autres départements et territoires français sont également le foyer de législations particulières en matière cultuelle. Pour 
diverses raisons, tenant notamment à la distance, tout l'Outre-mer ne s'est pas vu appliquer la loi du 9 décembre 1905. Cinq territoires sont régis par les décrets Mandel, adoptés en 1939 et entrés en vigueur à des dates différentes, en raison de la seconde guerre mondiale : la Polynésie française (pour les catholiques car, pour les protestants, s'applique un décret du 5 juillet 1927), la Nouvelle-Calédonie (où les décrets Mandel sont appliqués depuis 1943), les îles Wallis-et-Futuna (depuis 1948), Saint-Pierre-et-Miquelon (depuis 1956) et Mayotte (pour les catholiques car le culte musulman, qui y est majoritaire, se voit appliquer un droit local particulier). En Guyane française, le régime en vigueur repose sur une ordonnance royale du 27 août 1828 , comme l'a rappelé l'arrêt Beherec $^{17}$ du Conseil d'État: seul le culte catholique est envisagé et le clergé catholique, agréé comme ministre du culte par arrêté préfectoral, est rémunéré par le département.

Or, en se référant à la volonté des constituants, le Conseil ne justifie ici que l'existence du droit local alsacien-mosellan. Les autres régimes spécifiques ne trouvent aucune assise dans la présente décision. Leur position est même fragilisée dans la mesure où ils se trouvent tous en contradiction avec la consistance donnée par le Conseil au concept même de laïcité. Faut-il alors recourir à des justifications différentes pour chacun de ces régimes, la volonté des constituants dans un cas, l'histoire dans un autre, la distance géographique dans un troisième, la présence d'un nombre plus important de fidèles de telle ou telle confession ailleurs, etc. ? Ou bien le Conseil pourrait-il fonder ces législations spécifiques sur l'article 73 de la Constitution qui, s'il pose le principe que les lois et règlements nationaux s'appliquent de plein droit dans les départements et régions d'Outre-mer, admet qu'ils puissent « faire l'objet d'adaptations tenant aux caractéristiques et contraintes particulières de ces collectivités "?

3) De plus, l'argument des travaux préparatoires des constituants semble ici particulièrement dévoyé. L'utilisation qui en est faite est inédite. En l'occurrence, le Conseil invoque ces travaux préparatoires pour faire échec à l'application d'une disposition constitutionnelle, le principe constitutionnel de laïcité. «L'exception à cette interdiction [de rémunération des cultes] dégagée par le Conseil résulte seulement de l'analyse des travaux préparatoires. Puisque la règle découle du texte de la Constitution, l'exception n'aurait-elle pas dû elle aussi résulter d'une disposition constitutionnelle explicite? Le recours aux travaux

17. CE, 9 octobre 1981, Beherec, $\mathrm{n}^{\circ} 18649$. 
préparatoires est traditionnellement admis pour éclairer le sens d'un texte ou orienter son interprétation, non pour y ajouter des règles qui n'y figurent pas ${ }^{18}$. $»$ Est-il vraiment admissible d'adjoindre les travaux des constituants au bloc de constitutionnalité ? Car, ce n'est ni plus ni moins ce que fait ici le Conseil puisqu'il justifie la non-application d'un principe de valeur constitutionnelle en ayant recours à ces travaux préparatoires. Ceux-ci auraient certes pu éclairer légitimement l'interprétation de la Constitution, mais ils ont ici joué un rôle beaucoup plus central : ils ont permis de ne pas appliquer le principe constitutionnel de laïcité, ce qui est hautement contestable.

4) Enfin, le Conseil constitutionnel contourne soigneusement le problème soulevé par la requête. L'association requérante soulevait la méconnaissance du principe de laïcité. Or la motivation de la décision n'apporte aucune réponse à cet argument. Le contrôle de l'abrogation implicite ici effectué se contente de prendre note de la volonté des constituants, mais ne s'intéresse nullement à la conciliation de la norme législative contrôlée avec la norme constitutionnelle de référence, ce qu'aurait pourtant impliqué un contrôle de l'abrogation tacite pleinement effectué. La constitutionnalité des normes législatives se trouve donc assénée sans que le contrôle mis en œuvre ait été correctement exercé, ni que le problème central ait été abordé.

Il faut donc dresser un constat qui n'a hélas rien de neuf. Cette décision du Conseil, à l'instar de certaines de ses devancières, pèche par la faiblesse de son argumentation. «Comment ne pas critiquer la méthode employée dans ce jugement constitutionnel ? La décision du Conseil est courte, elle ne dit (presque) rien; elle constate puis tranche, sans justifier. Elle se contente tout d'abord d'opérer les constats suivants: existence d'un corpus législatif et réglementaire maintenant un régime dérogatoire en Alsace-Moselle... existence d'un corpus constitutionnel s'imposant au législateur... existence de travaux préparatoires des Constitutions de 1946 et 1958 ne remettant pas en cause les dispositions particulières visées. Conclusion, aussi rapide que non explicitée : le grief tiré de la violation du principe de laïcité doit être écarté ${ }^{19}$.»

18. Jean-François Amédro, «Le Conseil constitutionnel et la laïcité. À propos de la décision Association pour la promotion et l'expansion de la laïcité du Conseil constitutionnel ", JCP A, 2013, $\mathrm{n}^{\circ} 12$, act. 243.

19. Franck Laffaille, «La neutralisation du principe de laïcité au profit du droit culturel alsacien mosellan - À propos d'une décision... dénuée de fondements juridiques pertinents ", JCP A, 2013, n 16, p. 2108. 
Cette carence de motivation doit dès lors nous inciter à rechercher un fondement plus solide au maintien de ces dispositions législatives particulières à l'Alsace-Moselle.

\section{La recherche d'un fondement plus solide au maintien du droit local : critique de la définition de la laïcité retenue par le Conseil constitutionnel}

"Le régime dérogatoire alsacien-mosellan peut juridiquement être défendu. Encore faut-il tenter de trouver des fondements crédibles ${ }^{20}$." Or la décision APPEL n'assure pas au droit local les fondements capables d'en justifier l'existence. Elle laisse notamment irrésolu le problème de la conciliation du principe constitutionnel de laïcité et de la législation relative à la rémunération des ministres du culte en Alsace-Moselle. La méthode retenue révèle d'ailleurs la volonté du Conseil constitutionnel d'éluder cette question que les requérants avaient pointée du doigt. Aussi voulons-nous essayer ici de justifier plus solidement le maintien du droit local des cultes, en abordant ce problème sans détours.

Selon nous, l'apparente non-conformité du principe de laïcité et des dispositions locales relatives à la rémunération des ministres du culte provient de la définition que le Conseil constitutionnel donne du principe constitutionnel de laïcité et qui assimile ce principe constitutionnel et la loi du 9 décembre 1905 (a). Cette non-conformité peut être résorbée si l'on opère, comme nous y invite le droit positif $(b)$, une disjonction du principe constitutionnel de laïcité et de la loi de 1905 (c). Car il faut bien comprendre l'objet de la solution proposée: il ne s'agit pas de critiquer la définition de la laïcité donnée par le Conseil sur le fondement d'une approche doctrinale, mais de dénoncer ses contradictions avec le droit positif.

\subsection{La définition de la laïcité du Conseil constitutionnel: l'assimilation du principe constitutionnel de laïcité et de la loi du 9 décembre 1905}

1) Le considérant 5 de la décision APPEL définit le principe constitutionnel de laïcité par plusieurs composantes. Est inclue en premier lieu «la neutralité de l'État» qui correspond, d'une part, à la nonconfessionnalité de l'État, l'État ne devant prendre parti pour aucune religion en particulier, et, d'autre part, à la non-discrimination des 
citoyens pour des motifs de croyances religieuses. De ce double aspect de la neutralité de l'État, le juge déduit, d'une part, que «la République ne reconnaît aucun culte» (non-confessionnalité de l'État) et, d'autre part, «que le principe de laïcité impose notamment le respect de toutes les croyances, l'égalité de tous les citoyens devant la loi sans distinction de religion et que la République garantisse le libre exercice des cultes » (non-discrimination des citoyens); l'emploi du notamment indique en outre que cette approche du principe de laïcité n'est pas limitative ${ }^{21}$. En dernier lieu, le Conseil constitutionnel ajoute que la République «ne salarie aucun culte».

L'inspiration de ces différentes composantes n'est point douteuse: elle dérive indéniablement de la loi du 9 décembre 1905 concernant la séparation des Églises et de l'État, et notamment de ses articles $1^{\mathrm{er}}$ et 2. Conservant au principe de laïcité son unité notionnelle ainsi que toute sa charge idéologico-historique, cet aspect de la solution a pu être salué : « en consacrant l'idée selon laquelle le principe constitutionnel de laïcité puise une bonne partie de son contenu dans la loi de Séparation, plus précisément dans son titre I, le Conseil a adopté une solution symboliquement importante et historiquement pertinente car les principes et l'esprit de la laïcité trouvent leur formulation juridique la plus aboutie dans la loi de Séparation. Dissocier nettement la "laïcité législative" de la "laïcité constitutionnelle" aurait affaibli la notion de laïcité en limitant son contenu constitutionnel à l'affirmation de la liberté religieuse et d'un vague principe de neutralitée 22 .»

2) Mais ces considérations suffisent-elles à établir la pertinence juridique de l'interprétation délivrée par le Conseil ? Plus précisément, que faut-il penser de l'inclusion de ces quatre points dans la définition de la laïcité?

Le principe de neutralité de l'État ne pose guère de problème. Il forme indéniablement le noyau dur de la laïcité. Le problème demeure de savoir ce qu'est cette neutralité. S'agit-il d'une neutralité maximaliste, c'està-dire l'abstention de l'intervention de l'État dans le domaine religieux? Ou bien cette neutralité doit-elle recevoir une acception plus minimaliste et être alors conçue comme la possibilité d'une intervention de l'État dans le domaine religieux, sans que cette intervention se fasse en faveur

21. "Comme l'indique le "notamment", il ne s'agit pas d'une définition limitative du principe constitutionnel de laïcité, mais d'une énumération des règles essentielles qu'il impose et qui peuvent se concilier entre elles. ", commentaire de la décision aux Cahiers du Conseil constitutionnel, p. 20.

22. Jean-François Amédro, art. cité. 
ou en défaveur d'un culte particulier? En incluant dans la laïcité la nonreconnaissance des cultes et l'interdiction de les salarier, il semble que le Conseil retienne la conception maximaliste de la neutralité de l'État.

Le Conseil constitutionnel rattache également à la laïcité la liberté religieuse. L'affirmation de ce principe est facilement admissible. La liberté religieuse est le droit des citoyens corrélatif à l'obligation de neutralité de l'État: c'est parce que l'État reste neutre que les citoyens disposent de la liberté religieuse; ou, formulé autrement, c'est pour que la liberté religieuse des citoyens soit préservée que l'État doit demeurer neutre. Sur ce point, le principe constitutionnel de laïcité renvoie clairement à l'article 10 de la Déclaration des droits de l'homme et du citoyen, qui consacre la liberté de conscience. Ce principe est donc doté d'une valeur constitutionnelle évidente. Il est en outre rappelé par l'article $1^{\mathrm{er}}$ de la loi de 1905, qui précise que «la République assure la liberté de conscience. Elle garantit le libre exercice des cultes ».

Ayant de la neutralité de l'État une approche maximaliste, la décision $A P P E L$ intègre à la notion de laïcité la non-reconnaissance des cultes et l'interdiction de leur financement par l'État ${ }^{23}$. Ce double principe puise également son inspiration dans la loi du 9 décembre 1905 et, plus spécialement, dans son article 2, qui dispose que «la République ne reconnait, ne salarie ni ne subventionne aucun culte ». Ce faisant, le Conseil ne fait que reprendre une opinion doctrinale courante: "souvent regardée comme la source matérielle de la notion ${ }^{24}$ », la loi du 9 décembre 1905 serait l'expression unique du principe de laïcité. C'est ce qu'exposait sans ambages une réponse du ministre des départements et territoires d'Outre-mer à la question écrite d'un député de Walliset-Futuna: «Les principes posés par la loi du 9 décembre 1905 doivent être considérés comme "principes fondamentaux reconnus par les lois de la République" en ce qu'ils précisent le principe constitutionnel de la laïcité de la République française rappelé par l'article 2 [actuel article $1^{\text {er }}$ ] de la Constitution du 4 octobre 1958. Tel est le cas des principes de liberté de conscience, de libre exercice des cultes et d'interdiction de

23. «Les deux règles entretiennent entre elles un rapport de cause à effet : l'interdiction faite aux collectivités publiques de financer les cultes constitue l'un des moyens permettant une mise en œuvre effective de leur non-reconnaissance. », dans Mylène Le Roux, «La règle d'abstention financière des pouvoirs publics en matière cultuelle», $R D P, 2007, \mathrm{n}^{\circ} 1$, p. 266-267.

24. Jean-Pierre Machelon, «La laïcité : tradition et innovation », dans Bertrand Mathieu (dir.), 1958-2008: Cinquantième anniversaire de la Constitution française, Paris, Dalloz, Association française de droit constitutionnel, 2008, p. 183. 
subventionnement des cultes par l'État, le département et les communes, énoncés par les articles $1^{\text {er }}$ et 2 de la loi du 9 décembre $1905^{25}$."

Rattachés au principe de neutralité dans le sens maximaliste que semble lui conférer ici le Conseil, ces deux principes posent d'évidents problèmes au regard de la réglementation d'Alsace-Moselle. Dans les départements de Moselle, du Haut-Rhin et du Bas-Rhin, sont reconnus certains cultes (catholique, israélite, luthérien et calviniste); la reconnaissance de ces cultes permet leur subventionnement et notamment, comme l'exposait le cas d'espèce, la rémunération des ministres du culte. L'incompatibilité est flagrante: le considérant 5 de la décision affirme que la laïcité implique que la République "ne salarie aucun culte", tandis que la loi de l'an $\mathrm{X}$ permet de rémunérer les ministres du culte. A priori donc, cette disposition législative est en opposition avec le principe constitutionnel de laïcité, auquel le Conseil intègre la substance des articles $1^{\text {er }}$ et 2 de la loi de 1905. Mais comme le Conseil entend maintenir en vigueur la législation locale, on peut se demander s'il était pertinent d'assimiler aussi radicalement le principe constitutionnel de laïcité et la loi du 9 décembre 1905. Interrogeons le droit positif sur ce point.

\subsection{Le refus du droit positif d'assimiler purement et simplement le principe constitutionnel de laïcité et la loi du 9 décembre 1905}

Que faut-il penser de l'assimilation que fait le Conseil constitutionnel entre le principe constitutionnel de laïcité et la loi du 9 décembre 1905 ? Il est certain que la loi de 1905 consacre certains principes évidemment rattachables à la laïcité : ainsi en est-il de la neutralité de l'État et de la liberté religieuse. Néanmoins, un tel constat n'induit pas nécessairement l'assimilation de l'ensemble de cette loi au principe constitutionnel de laïcité. La question se pose notamment pour les principes de nonreconnaissance des cultes et de non-subventionnement par l'État, à qui l'article 2 de la loi de 1905 confère une indéniable portée législative. Le droit positif confirme-t-il l'intégration de l'article 2 de la loi de 1905 au principe constitutionnel de laïcité ?

1) Sans même revenir sur les législations cultuelles spécifiques à l'outre-mer, on relève que de nombreux dispositifs législatifs, qui ne

25. Réponse du ministre des Départements et Territoires d'Outre-Mer à la question écrite $\mathrm{n}^{\mathrm{O}} 20155$, JO, 13 novembre 1995, p. 4827. 
peuvent pas tous se targuer d'une spécificité historique (comme en Alsace-Moselle), dérogent à l'article 2 de la loi de 1905.

La loi de 1905 admet elle-même des dérogations à la règle qu'elle pose. Le même article 2 permet l'inscription aux budgets des personnes publiques des « dépenses relatives à des services d'aumônerie et destinées à assurer le libre exercice des cultes dans les établissements publics tels que lycées, collèges, écoles, hospices, asiles et prisons ». L'article 13 de la loi de 1905 dispose quant à lui que «l'État, les départements, les communes et les établissements publics de coopération intercommunale pourront engager les dépenses nécessaires pour l'entretien et la conservation des édifices du culte dont la propriété leur est reconnue par la présente loi ».

D'autres lois ont apporté des dérogations, d'importance variable, à l'article 2 de la loi de 1905. Ainsi par exemple, les associations et congrégations religieuses bénéficient d'exonérations fiscales diverses, ce qui s'assimile bel et bien à un financement négatif, puisque l'État leur abandonne une part des recettes qu'il pourrait exiger d'elles: on peut citer à ce titre le fait que les édifices affectés à l'usage des cultes sont exonérés de la taxe foncière et de la taxe d'habitation ${ }^{26}$ ou le fait que les associations cultuelles sont dispensées du paiement des droits d'enregistrement pour les dons et legs ${ }^{27}$. De même, les communes et les départements sont autorisés à "garantir les emprunts contractés pour financer, dans les agglomérations en voie de développement, la construction, par des groupements locaux ou par des associations cultuelles, d'édifices répondant à des besoins collectifs de caractère religieux ${ }^{28} »$. On ne peut aucunement nier qu'il s'agit là « d'une dérogation majeure à la loi de 1905, puisqu'en cas de carence de l'association ou du groupement en cause, c'est la collectivité qui, de fait, assure le remboursement de l'emprunt qui a servi à financer la construction du lieu de culte ${ }^{29}$ ».

Si elles trouvent différentes justifications ${ }^{30}$, ces quelques illustrations montrent que le droit positif n'admet pas uniment le principe de nonsubventionnement des cultes. La règle d'abstention financière n'est pas

26. Article $1382,4^{\circ}$ du Code général des impôts.

27. Article $795,10^{\circ}$ du Code général des impôts.

28. Articles L 2252-4 et L 3231-5 du Code général des collectivités territoriales.

29. Édouard Geffray, "Loi de 1905 et aides des collectivités publiques aux cultes. Conclusions sur CE, 19 juillet 2011, $\mathrm{n}^{0}$ 308544, Trélazé (Cne)», RFDA, 2011, p. 967.

30. «Les dérogations à la règle d'abstention financière témoignent, d'une part, du souci d'assurer la conservation du patrimoine cultuel en permettant la prise en charge par les personnes publiques des dépenses d'entretien, de conservation ou de réparation des édifices culturels. Elles traduisent, d'autre part, la volonté du législateur de garantir la liberté d'exercice des cultes en la conciliant avec le principe de séparation. », dans Mylène Le Roux, art. cité. 
réduite à rien puisque le Conseil d'État veille toujours à son application rigoureuse quand la loi de $1905 \mathrm{~s}^{\prime}$ applique ${ }^{31}$. Mais il paraît contestable de lui conférer un rang constitutionnel, en l'intégrant à la notion de laïcité consacrée à l'article $1^{\text {er }}$ de la Constitution. "Il existe incontestablement un lien privilégié entre la laïcité et la suppression de toute dépense publique relative au culte. Ce lien, pour autant, n'est pas systématique; si bien que la valeur constitutionnelle du principe de laïcité ne peut être étendue à la règle d'abstention financière ${ }^{32}$. » Il faut sans doute davantage lui reconnaître une simple valeur législative, puisqu'il est consacré par la loi de 1905. Et l'on peut alors admettre que d'autres lois puissent, en Alsace-Moselle ou ailleurs, prévoir d'autres modalités d'application du principe de laïcité, qui permettent une forme de subventionnement des cultes, dans le respect d'une neutralité étatique comprise de manière moins maximaliste. Sinon, si l'on admet la valeur constitutionnelle du principe de non-subventionnement, toutes les dérogations législatives seraient inconstitutionnelles.

2) La jurisprudence administrative montre également que les principes de non-reconnaissance des cultes et de non-subventionnement par l'État, consacrés par l'article 2 de la loi de 1905, ne sont pas d'application uniforme et peuvent parfaitement recevoir des exceptions.

Dans l'important arrêt $S N E S^{33}$, le Conseil d'État a reconnu au principe de laïcité la valeur de PFRLR, sur le fondement de la loi de 1905. Or, pour qu'un PFRLR soit dégagé, il faut que son application n'ait subi aucune exception depuis sa consécration par une loi de la République antérieure à $1946^{34}$. Si l'article 2 de la loi de 1905 était intégré au principe de laïcité, les exceptions prévues à cet article par des lois postérieures seraient des exceptions au principe de laïcité lui-même. Sa qualification de PFRLR serait alors impossible. Or, puisqu'il y a PFRLR, il faut conclure que les exceptions à l'article 2 de la loi de 1905 ne constituent pas des exceptions à la laïcité elle-même. Par conséquent, pour le Conseil d'État, l'article 2 n'est pas assimilable au principe de laïcité.

Le Conseil d'État livre une interprétation identique et plus claire encore dans un arrêt Ministre de l'Outre-Mer. Il y affirme que «le

31. CE, sect., 9 octobre 1992, Commune de Saint-Louis de La Réunion, $\mathrm{n}^{\circ} 94455$. Pour une illustration récente: CE, 15 février 2013, Association Grande confrérie de SaintMartial, $\mathrm{n}^{\circ} 347049$.

32. Mylène Le Roux, art. cité.

33. CE, 6 avril 2001, Syndicat national des enseignements du second degré, dit arrêt SNES, $\mathrm{n}^{\text {os }} 219379,221699$ et 221700 .

34. CC, décision $n^{\circ} 88-244$, DC du 20 juillet 1988, Loi portant amnistie, considérants 11 et 12 . 
principe constitutionnel de laïcité qui s’applique en Polynésie française et implique neutralité de l'État et des collectivités territoriales de la République et traitement égal des différents cultes, n'interdit pas, par lui-même, l'octroi dans l'intérêt général et dans les conditions définies par la loi, de certaines subventions à des activités ou des équipements dépendant des cultes; que la loi du 9 décembre 1905 de séparation des églises et de l'État dont l'article 2 dispose que la République ne reconnaît, ne salarie ni ne subventionne aucun culte, n'a pas été rendue applicable en Polynésie française ${ }^{35}$ ». Le Conseil d'État admet simultanément que le principe de laïcité s'applique en Polynésie française, tandis que l'article 2 de la loi de 1905 y est quant à lui inapplicable : la dissociation du principe constitutionnel et de l'article législatif est évidente. L'interdiction de subventionnement des cultes n'a donc pas valeur constitutionnelle, mais simplement valeur législative ${ }^{36}$. "Le juge administratif put alors distinguer les effets du principe constitutionnel de laïcité, et ceux de la loi de 1905. Le premier s'applique en Polynésie, la seconde n'y a jamais été introduite; l'interdiction de subventionner un culte est une exigence législative, et non constitutionnelle. Les deux textes n'ont pas le même contenu: la laïcité constitutionnelle implique à la fois la neutralité de la République et l'égalité de tous les cultes. C'est donc une interprétation minimaliste et souple qui est encore donnée aux dispositions constitutionnelles ${ }^{37}$.»

Muni de ce constat législatif et jurisprudentiel, quelle conclusion peut-on tirer?

\subsection{Une interprétation de la laïcité plus en accord avec le droit positif : la nécessaire disjonction du principe constitutionnel de laïcité et de la loi du 9 décembre 1905}

Ce bref panorama indique suffisamment que le droit positif n'admet pas uniment les principes de non-reconnaissance des cultes et de nonsubventionnement par l'État. L'article 2 de la loi de 1905 qui les prévoit subit de bien nombreuses exceptions. Or, consacré par l'article $1^{\text {er }}$ de

35. CE, 16 mars 2005, Ministre de l'Outre-Mer, $\mathrm{n}^{\circ} 265560 ; A J D A, 2005$, p. 1463, note Claude Durand-Prinborgne.

36. «L'interdiction de subventionner un culte sur fonds publics est une exigence de rang législatif et non constitutionnel. Les textes en présence n'ont donc pas le même contenu. », Olivier Dord, "L'affirmation du principe constitutionnel de laïcité », dans Denys de Béchillon (dir.), L'architecture du droit. Mélanges en l'honneur de Michel Troper, Paris, Economica, 2006, p. 416.

37. Clément Benelbaz, Le Principe de laïcité en droit public français, L'Harmattan, coll. «Logiques juridiques», 2011, p. 132. 
la Constitution, le principe de laïcité est nécessairement d'application générale sur le territoire de la République, une et indivisible. La loi de 1905 s'avère en conséquence insusceptible de rendre compte, à elle seule, du principe constitutionnel de laïcité.

C'est pourquoi, pour fournir de la laïcité une interprétation plus en accord avec le droit positif, il s'avère nécessaire d'opérer une nette dissociation du principe de laïcité, de valeur constitutionnelle, et de l'article 2 de la loi de 1905, de valeur législative. Comme l'expose Jean-Marie Woehrling, «l'importance attribuée aux règles figurant à cet article $2 \mathrm{a}$ conduit parfois à y voir un principe de caractère constitutionnel. [...] Aujourd'hui cependant, cette conception paraît devoir être abandonnée : l'article 2 n'a qu'une valeur législative, d'ailleurs de portée incertaine ${ }^{38}$ ". En un mot, la laïcité n'est pas tout entière contenue dans la loi de 1905 ; le principe constitutionnel de laïcité peut faire l'objet d'une interprétation législative différant de celle effectuée en 1905. L'étude sommaire du droit positif à laquelle nous nous sommes livrés l'atteste : la majorité du territoire français est soumis à la loi de 1905, interprétation principale de la laïcité à la française; d'autres dispositifs législatifs peuvent néanmoins en prévoir une autre interprétation qui, différente de celle faite par la loi de 1905 , n'en demeure pas moins conforme à l'article $1^{\text {er }}$ de la Constitution.

1) Cette disjonction des principes constitutionnels et législatifs implique que la neutralité de l'État est entendue, non de manière maximaliste comme le fait le Conseil constitutionnel, mais de façon plus minimaliste. Quelles différences opposent ces deux acceptions de la neutralité de l'État?

Dans la vision minimaliste, «est neutre un État qui est aconfessionnel qui n'a pas par lui-même d'opinion religieuse particulière et ne soutient ni ne combat aucune idée religieuse déterminée. L'État n'a pas d'objectif religieux et ne doit pas avoir de motivation religieuse. Il ne doit pas non plus avoir des finalités religieuses ou des mobiles religieux ${ }^{39}$ ». Tant qu'il n'a pas d'opinion ou de motivation religieuses, l'État peut intervenir dans la sphère du religieux sans que sa neutralité en soit atteinte. L'idée générale est qu'il lui est impossible de faire abstraction du fait religieux, fait social trop important pour être ignoré du pouvoir politique. Dès lors, la neutralité de l'État s'accommode, non pas d'une confusion

38. Jean-Marie Woehrling, "L'interdiction pour l'État de reconnaître et de financer un culte. Quelle valeur juridique aujourd'hui ?», RDP, 2006, nº 6, p. 1633.

39. Jean-Marie Woehrling, "Le droit local alsacien-mosellan des cultes après les récentes décisions du Conseil constitutionnel ", $R D P, 2013, \mathrm{n}^{\circ} 3, \mathrm{p} .552$. 
du religieux et du séculier, mais d'une intervention de l'État dans le domaine religieux, pour des motifs d'intérêt général et dans le respect de la plus stricte impartialité, c'est-à-dire en traitant semblablement les cultes placés dans des situations semblables. Selon cette approche, l'État peut par exemple apporter des subventions à un culte si, dans le respect du principe d'égalité, des conditions objectives justifient que cette intervention financière réponde à un motif d’intérêt général.

Le raisonnement est inverse à celui tenu en 1905, où la neutralité de l'État était comprise de façon maximaliste. Cette acception, dont il n'est pas nécessaire de rappeler les liens avec l'anticléricalisme de la $\mathrm{III}^{\mathrm{e}}$ République, repose sur un postulat bien simple: le fait religieux doit être exclu de l'espace public et être cantonné à la sphère privée ${ }^{40}$. Il résulte de la séparation radicale du temporel et du spirituel une séparation tout aussi tranchée entre l'État, a priori étranger à la question religieuse, et les Églises, dont l'empire ne doit s'exercer que sur les consciences (celles qui l'acceptent bien sûr). Dans cette optique, la neutralité de l'État est définie comme une indifférence complète vis-à-vis du fait religieux, ignoré par le pouvoir politique puisque ne ressortant pas de son domaine de compétence. Cette conception impose donc à l'État, pour demeurer neutre, de ne reconnaître et de ne subventionner aucun culte, sans quoi il s'immiscerait dans un domaine sur lequel il n'a aucun avis à émettre: "C'est en ne reconnaissant, ne salariant ni ne subventionnant aucun culte, en ne privilégiant aucune croyance, en ne conférant aucun statut particulier aux églises que la République peut être neutre ${ }^{41}$.»C'est pourquoi l'acception maximaliste de la neutralité de l'État se rattache à la loi de séparation des Églises et de l'État. Si l'on juge la décision du Conseil constitutionnel à l'aune de cette conception, comme le fait Frédérique de La Morena, on peut déplorer que le Conseil constitutionnel n'ait consacré que quelques-uns des principes de la loi

40. «Juridiquement, la sphère publique concerne l'ensemble de la nation et a pour objet ce qui est universellement partagé; la sphère privée quant à elle est celle des individus et des communautés, libres dans le respect de la loi. L'indépendance de ces deux sphères est garantie par l'État qui se refuse, d'un côté, à imposer une doctrine, une croyance particulière et qui incarne, d'un autre côté, l'unité de la communauté politique et en promeut les valeurs communes. » (Frédérique de La Morena, «La laïcité: ni chiffon rouge, ni voile opaque ", tribune publiée dans Le Monde.fr, 27 mars 2012, disponible sur http://www.hci.gouv.fr/IMG/pdf/Tribune_F-de_la_Morena_Le_Monde-fr-2.pdf, consulté le 18/11/2013).

41. Frédérique de La Morena, «Laïcité de la République et droit local, une construction constitutionnelle fragile - À propos de la décision $n^{0}$ 2012-297 QPC du 21 février 2013 ", Droit administratif, 2013, $n^{\circ} 8$, étude 14. 
de séparation, et non pas tous ${ }^{42}$, et ait jugé constitutionnel le droit local des cultes ${ }^{43}$.

Des deux définitions possibles de la neutralité de l'État, le droit positif retient pourtant l'acception minimaliste. En distinguant principe constitutionnel de laïcité et loi de 1905, il n'assimile pas la neutralité de l'État, évidemment comprise dans le principe constitutionnel de laïcité, et la séparation des Églises et de l'État, de laquelle ressortent les interdictions de reconnaissance et de subventionnement des cultes. La laïcité constitutionnelle "implique une séparation du politique et du religieux que traduit une neutralité confessionnelle de l'État proche de l'impartialité. [...] Cette neutralité bienveillante n'interdit pas le financement public des cultes ou de l'enseignement confessionnel. En revanche le [principe législatif de séparation des cultes et de l'État] met fin au régime des cultes reconnus en supprimant la nomination par l'État des ministres du culte et le statut de droit public des Églises. Il prohibe sauf exception le financement public des cultes ${ }^{44}$ ». Plus encore, le principe de neutralité (constitutionnel) prime celui de séparation (législatif). Le premier est donc d'application uniforme et intangible sur le territoire national, tandis que le second n'est qu'une modalité possible de mise en œuvre du principe de neutralité et comprend diverses dérogations et aménagements.

Si l'on veut rendre objectivement compte du droit positif, on doit reconnaître que celui-ci consacre l'acception minimaliste du principe de neutralité puisqu'il rompt la nécessité du lien entre la neutralité de l'État et l'interdiction qui lui est faite de reconnaître et de subventionner les cultes. Il est envisageable de critiquer cette orientation du droit positif, mais le constat n'en demeure pas moins incontestable. Aussi, loin de

42. «La laïcité, en droit, est l'expression juridique d'une conception politique qui implique la séparation des églises et de l'État, afin de garantir la liberté de conscience et la liberté religieuse. Cette conception de la laïcité est le résultat d'une longue évolution historique qui s'achève avec sa consécration constitutionnelle, mais qui a été préparée antérieurement par de nombreux textes, particulièrement la loi dite "loi de séparation" dont le Conseil constitutionnel, discrétionnairement, consacre certains des principes qu'il associe à d'autres dispositions de la Constitution, sans même mentionner le principe de séparation de l'État et des églises et donc, sans présenter une interprétation complète du contenu de la laïcité. », Frédérique de La Morena, ibid.

43. «Les juges auraient pu conforter la laïcité constitutionnelle en allant jusqu'au bout d'une démarche consistant non seulement à intégrer tous les principes de 1905 mais encore à en rendre la lecture cohérente. Le statut des cultes d'Alsace-Moselle aurait alors été jugé inconstitutionnel. », Frédérique de La Morena, ibid.

44. Olivier Dord, art. cité, p. 418. 
considérer que la définition du Conseil constitutionnel s'éloigne trop de la loi de séparation, semble-t-il plutôt que la déficience première de la décision APPEL est de s'écarter de la réalité juridique, en optant pour une conception maximaliste de la neutralité étatique qui, inspirée de la loi de 1905, ne rend plus un compte exact du droit positif actuel.

2) L'application de ces principes au cas d'espèce montre tout l'intérêt de cette dissociation entre le principe de laïcité reconnu à l'article $1^{\mathrm{er}} \mathrm{de}$ la Constitution et la loi de 1905. Cette dissociation a la vertu de rendre conforme le principe constitutionnel de laïcité et le droit local des cultes. En effet, le principe de laïcité, consacrant l'approche minimaliste de la neutralité de l'État, ne s'oppose plus à la disposition législative ici déférée, qui permet une rémunération des ministres du culte.

Le droit local des cultes ne serait ainsi qu'un aménagement des rapports entre l'État et les cultes qui, pour être différent de celui prévu par la loi de 1905, n'en serait pas pour autant contraire au principe constitutionnel de laïcité. Il n'y a certes pas séparation des Églises et de l'État, mais le principe de laïcité n'est pas violé pour autant puisqu'une telle séparation n'est que l'une des modalités possibles de sa mise en œuvre. Si bien que la disposition en cause, et plus généralement le droit local des cultes, se conforment au principe de laïcité de la République et n'entravent pas la neutralité de l'État. "Ainsi le système alsacienmosellan de droit des cultes ne se perçoit pas comme à "l'extérieur" de la laïcité, mais comme une autre modalité de la laïcité ${ }^{45}$.» Tel est d'ailleurs ce qu'avait exprimé le Conseil d'État, dans son rapport annuel de 2004 : « on peut voir dans la situation prévalant en Alsace-Moselle une forme particulière de l'organisation des rapports et de la séparation des Églises et de l'État ${ }^{46}$.»

Ce raisonnement assure au droit local une plus grande pérennité dans la mesure où il le rend conforme au principe constitutionnel de laïcité. À l'inverse, en appuyant sa décision sur la seule volonté du constituant et non sur une norme constitutionnelle, le juge laisse tout loisir au législateur de modifier la législation locale ${ }^{47}$, ce qui la

45. Jean-Marie Woehrling, «Le droit local alsacien... », art. cité, p. 533.

46. "Un siècle de laïcité », EDCE, 2004, p. 269.

47. "Le Conseil constitutionnel n'élève pas le droit local des cultes au rang de norme constitutionnelle. Il est seulement jugé conforme à la Constitution parce que celle-ci n’a pas entendu "remettre en cause" les dispositions législatives ou réglementaires particulières applicables dans plusieurs parties du territoire de la République (ce qui vise l'Alsace-Moselle et les autres régimes des cultes dérogatoires à la loi de 1905). Le législateur pourrait donc en abroger les dispositions législatives. ", Elsa Forey, «Le Conseil constitutionnel au secours du droit local des cultes», art. cité. 
fragilise grandement. On peut donc conclure, avec Jean Morange ${ }^{48}$, que le Conseil constitutionnel aurait justifié sa décision de manière plus satisfaisante si, acceptant une vision plus minimaliste de la neutralité de l'État, il avait détaché le principe constitutionnel de laïcité de la loi de 1905, d'accord en cela avec le droit positif.

Doctorant en droit public université de Paris 1 - Panthéon-Sorbonne

48. «Il aurait été préférable d'atteindre le même résultat, garantir le maintien d'un régime spécifique à l'Alsace-Moselle, en raisonnant autrement... La définition et la portée du principe de laïcité aurait gagné à être énoncées le plus simplement possible à partir de la seule neutralité de l'État. » (Jean Morange, «Le "mystère" de la laïcité à la française ", $\left.R D P, 2013, \mathrm{n}^{\circ} 3, \mathrm{p} .518\right)$. 